Historic, Archive Document

Do not assume content reflects current scientific knowledge, policies, or practices. 



\subsection{3}

(1)

AURORA GROWN MEANS WELL GROWN

Spring 1920

Wholesale Trade List

\section{Aurora Nurseries}

Aurora Nursery Co., Prop.

\section{Aurora, Illinois}

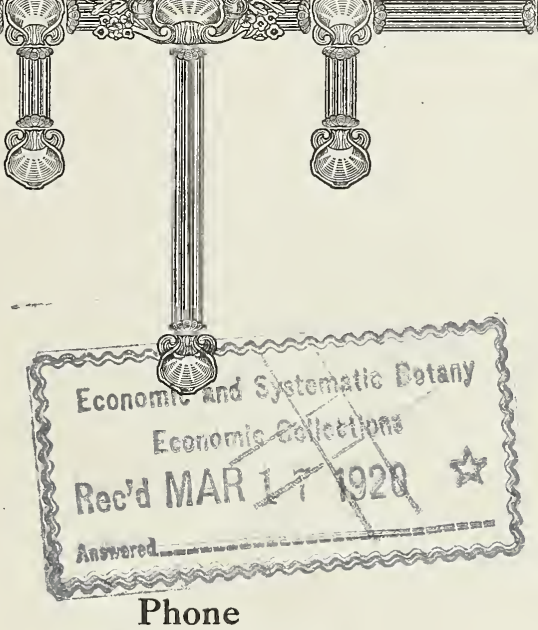

All Departments

339

Grown Right - Dug Right - Packed Right 


\section{THANK YOU}

1919 has passed forever, and the brighter days of 1920 invite us to renewed activities. It was a privilege to be intrusted with your orders during 1919. We hope we pleased you. We tried to render an honest service and we trust that our activities have not been a disappointment to you. You must be pleased with our service and our goods else we cannot prosper.

For 1920 we ask for your continued orders. Our stock shall be right. Our service quick and efficient and our prices as low as good plants should be sold.

To those who have never ordered from us, we ask that you give us a trial. We will try to give you quick shipment and honest goods at fair prices.

Remember, that Aurora products are Grown Right-Dug Right-Packed Right.

\section{TERMS, ETC.}

How to Ship-When ordering always specify whether we shall ship by freight or express. Also give us the route if possible. When no directions are given we will use our best judgment as to how to ship, but in no case will we be responsible after goods are consigned to purchaser. When losses or damage occur while goods are in transit, either from delay or otherwise, make claim at once to forwarding Companies.

Terms-Terms of payment are cash or security before shipment. Our usual terms of credit will be accorded to established firms, who if not known to us will please forward references. C. O. D. orders must be accompanied by one-fourth the amount of the order in cash.

Discounts-Three per cent discount is allowed on cash with order. Two per cent discount for cash in 10 days from date of invoice.

Prices-Prices are for the Spring 1920. This list supercedes all other lists and is subject to change without notice. Five plants of a variety will be supplied at the rate per ten-fifty at the rate per hundred-five hundred at the rate per thousand.

Claims-Claims for deduction must be made within one week after receipt of goods.

Packing-Prices in this list are Net; cost of Boxes, Bales, etc., will be added.

Guaranty-While we exercise the greatest care to have our trees and plants true to label, and hold ourselves prepared to replace, on proper proof, all that may prove untrue, we do not give any warranty expressed or implied, and in case of any error on our part, it is mutually agreed between the purchaser and ourselves that we shall not at any time be held responsible for a greater amount than the original price of the trees. lined.

Boxing Charges-Charges are made as low as possible for well made boxes, paper

$\begin{array}{ll}30 \times 30 \times 6 & \text { feet. } \ldots \$ 4.50 \\ 30 \times 30 \times 10 & \text { feet... } 5.00\end{array}$

$30 \times 30 \times 12$ feet... 5.50

$24 \times 30 \times 6$ feet.... 3.50

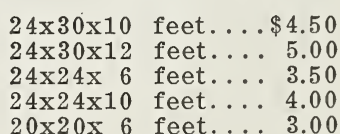

$20 \times 20 \times 10$ feet. . $\$ 3.50$

$18 \times 24 \times 6$ feet.... 3.00

$18 \times 18 \times 6$ feet.... 2.50

$18 \times 18 \times 10$ feet.... 3.00

\section{SEED CORN}

A few years ago we obtained two bushels of 90 day yellow corn. Ve found this to be a mighty fine kind. It was caid to have come from North East Nebraska. We received no name for it but simply call it North Fast Nebraska. This year this corn ripened in 84 days. We have about eight bushels, and if interested in something for your own use it is mighty fine. Price, 25 cents per pound, 10 pounds $\$ 2.25$. One-half bushel $\$ 7.00$, one bushel $\$ 12.50$.

\section{YOUNG STOCK FOR LINING OUT}

The list of small stocks is made up under a special circular and if interested will be glad to quote on what we can do. 


\section{DECIDUOUS TREES}

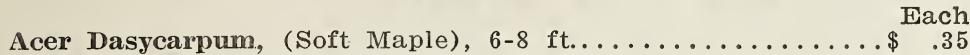

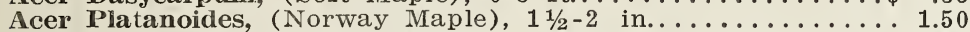

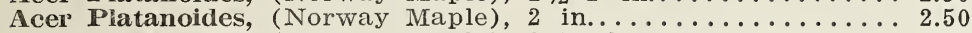

Acer Platanoides, (Norway Maple), $8-10$ ft. ....................

Acer Saccharinum, (Sugar Maple), $4-5 \mathrm{ft} . \ldots \ldots \ldots \ldots \ldots \ldots . . . .40$

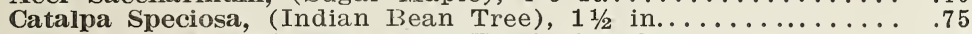

Catalpa Speciosa, (Indian Bean Tree), 6-8 ft.............40

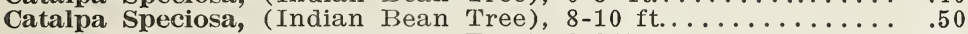

Catalpa Speciosa, (Indian Bean Tree), $2-2 \frac{1}{2}$ in............ 1.35

Catalpa Bungei, (Globe Catalpa), 3 yr. heads, 6-7 ft. stems... . 1.50

The Catalpa Bungei offered are top notchers. They are almost perfect and are well worth the price asked.

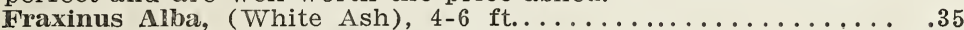

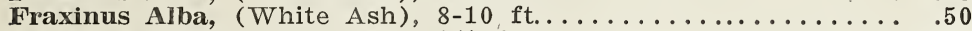

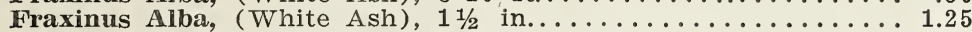

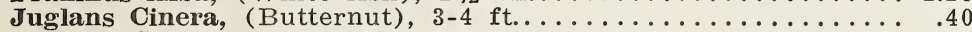

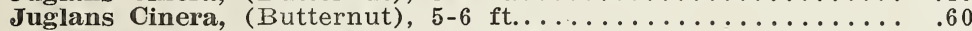

Morus Alba, (Russian Mulberry), $3-4 \mathrm{ft} . \ldots \ldots \ldots \ldots \ldots \ldots \ldots \ldots . \ldots . \ldots \ldots$

Populus Alba, (Silver Poplar), $4-5 \mathrm{ft} . \ldots \ldots \ldots \ldots \ldots \ldots \ldots \ldots . \ldots \ldots . \ldots \ldots$

Populus Alba, (Silver Poplar), $5-6 \mathrm{ft} \ldots \ldots \ldots \ldots \ldots \ldots \ldots \ldots \ldots \ldots$

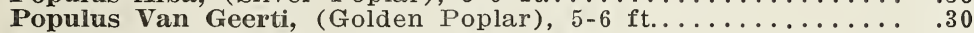

Prunus Serotina, (Wild Cherry), $8-10 \mathrm{ft} \ldots \ldots \ldots \ldots \ldots \ldots \ldots \ldots . \ldots . . \ldots$

Prunus Americana. (Wild Flum), $6-8 \mathrm{ft} \ldots \ldots \ldots \ldots \ldots \ldots \ldots \ldots \ldots . . . \ldots \ldots$

Quercus Palustrus, (Pin Oak), $3-4$ ft...................40

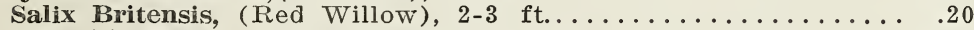

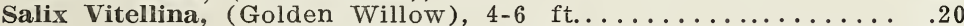

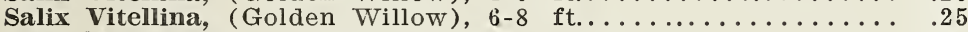

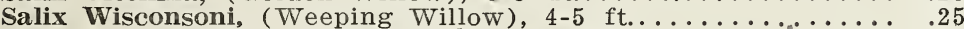

Salix Wisconsoni, (Weeping Willow), 3-4 ft..............20

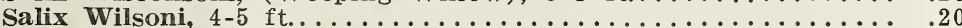

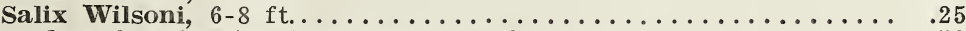

Sorbus Acquiparia, (ivt. Ash), $6-8 \mathrm{ft} \ldots \ldots \ldots \ldots \ldots \ldots \ldots \ldots \ldots . \ldots \ldots . \ldots \ldots$

Ulmus Americana. (American Elm), 6-8 ft...............40

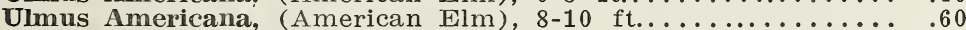

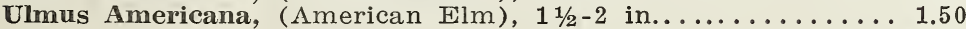

$10 \quad 100$

$\$ 3.00 \$ 25.00$

8.00

3.50

6.00

3.00

4.50

12.50

25.00

40.00

100.00

2.50

4.50

10.00

3.00

5.00

2.00

1.75

3.00

2.00

4.00

3.00

3.50

1.50

1.50

1.75

1.75

1.50

1.50

2.00

4.50

3.50

4.50

12.50

30.00

40.00

110.00

\section{WEEPING TREES}

Cut Leaf Weeping Birch, $5-6 \mathrm{ft} . \ldots \ldots \ldots \ldots \ldots \ldots \ldots \ldots \ldots . \ldots \ldots{ }_{85}$

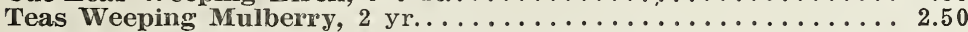

\section{SHRUBS}

Each

10

3.00

3.50

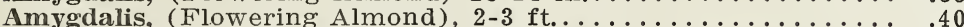

2.50

3.50

3.50

1.25

1.50

2.00

1.50

1.75

1.75

1.50

1.75

1.75

2.50

2. 00

2.00

7.50

1.75

4.50

6.00

4.50
100

32.00

8.00

12.50

17.50

12.00

15.00

12.00

15.00

15.00

17.50

30.00
Calycanthus $\mathbb{F l o r i d u s , ~ ( S w e e t ~ S c e n t e d ~ S h r u b ) , ~ 1 8 - 2 4 ~ i n . . . . . . . ~ . ~ . ~} 20$

Crataegus Crus Galli, (Cockspur Thorn), $3-4$ ft........... . .50

Crátaegus Crus Galli, (Cockspur Thorn), 4-5 ft............

BUTTERFLY BUSH, from 2 in. pots, delivery in May 15 cents each; $\$ 1.00$ per $10 ; \$ 8.00$ per 100 


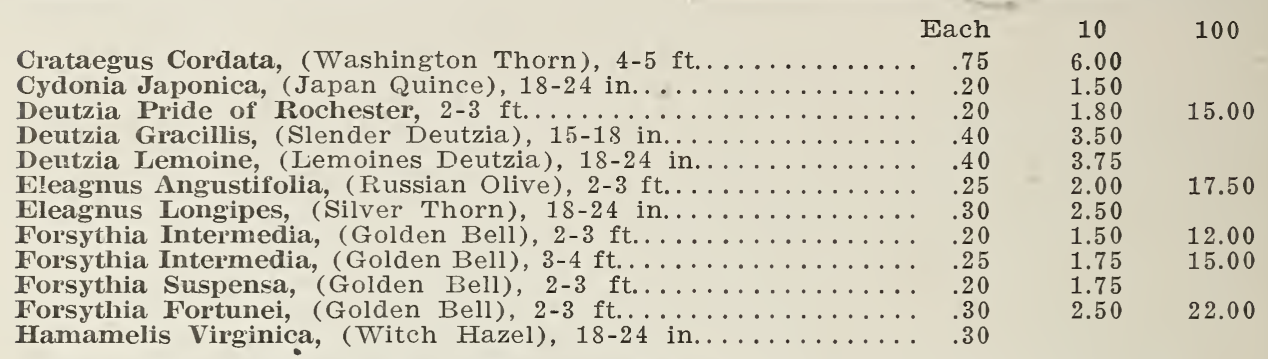

HYDRANGEA, P. G., 12-18 in.......\$.30 each; \$2.50 per 10;\$22.50 per 100 HYDRANGEA, P. G., 18-24 in.........40 each; 3.50 per 10; 30.00 per 100 HYDRANGEA, P. G., 2-3 ft.........4.45 each; 4.00 per 10; 35.00 per 100

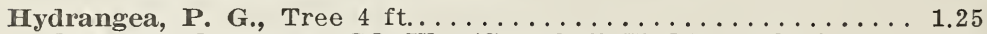

Hydrangea Aborescens Gd. Fl., (Snowball Hyd.), $18-24$ in.... 35

Hydrangea Arborescens Gd. Fl, (Snowball Hyd.), 2-3 ft. . . . . . . . .50

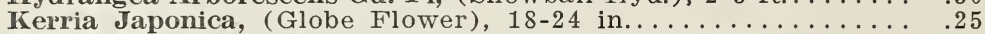

Kerria Japonica Fl. Pl., (Dbl. Globe Flower), 18-24 in....... .25

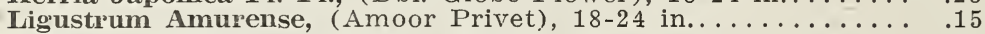

Ligustrum Amurense, (Amoor Privet), 2-3 ft.............20

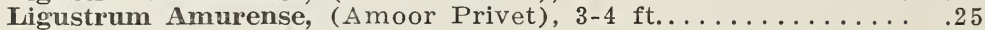

Iigustrum Ibota, (Ibota Privet), $12-18$ in................15

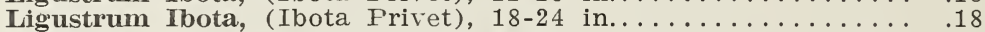

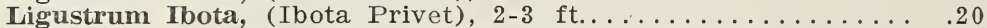

Ligustium Leucocarpum, (White Fruited), $2-3 \mathrm{ft} . \ldots \ldots \ldots . . .25$

Lonicera Morpowi, (Morrows Honeysuckle), $2-3 \mathrm{ft} . \ldots \ldots \ldots . . . .25$

Lonicera Morrowi, (Morrows Honeysuckle), $3-4 \mathrm{ft} \ldots \ldots \ldots \ldots . . . .35$

Lonicera Grandiflora, (Pink H. S.), $2-3 \mathrm{ft} . \ldots \ldots \ldots \ldots \ldots \ldots . . \ldots \ldots$

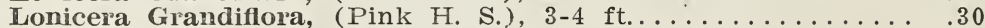

Lonicera Bella Albida, (Upright White $\mathrm{H}$. S.), $3-4 \mathrm{ft} \ldots \ldots \ldots \ldots .25$

Morus Alba, (Russian Mulberry), $3-4 \mathrm{ft} \ldots \ldots \ldots \ldots \ldots \ldots \ldots \ldots \ldots . . \ldots \ldots$

Philadelphus Coronarius, (Mock Orange), $2-3 \mathrm{ft} . \ldots \ldots \ldots . \ldots . . . .20$

Philadelphus Coronarius, (Mock Orange), 3-4 ft........... .25

Philadelphus Gd. Fl., (Large Flower Syr.), $3-4 \mathrm{ft} . \ldots \ldots \ldots \ldots . . . .20$

Philadelphus Gd. Fl., (Large Flower Syr.), 4-5 ft......... .25

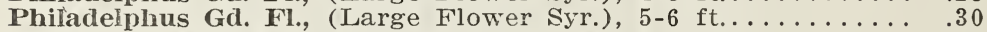

Philadelphus Nivalis, (Snowdrift Syr.), 3-4 ft............ .25

Philadelphus Nivalis, (Snowdrift Syr.), $4-5 \mathrm{ft} . \ldots \ldots \ldots \ldots \ldots$

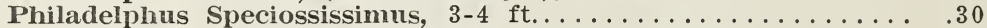

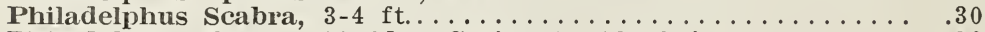

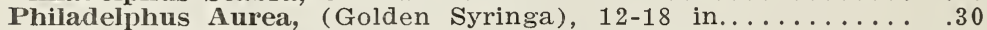

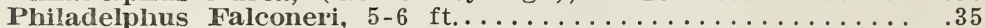

Prunus Besseyi, (Dwf. Rocky it. Cherry), $2-3 \mathrm{ft} \ldots \ldots \ldots . .35$

Prunus Pumila, (Sand Cherry), $2-3 \mathrm{ft} \ldots \ldots \ldots \ldots \ldots \ldots \ldots \ldots . \ldots \ldots$

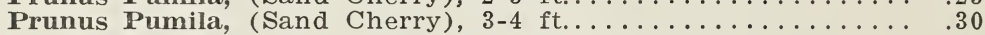

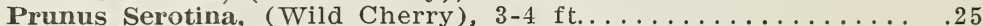

Prunus Serotina, (Wild Cherry), $5-6$ ft. . . . . . . . . . . . . .

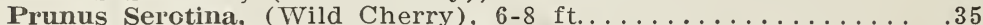

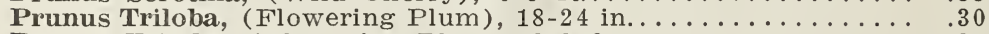

Prunus Triloba, (Flowering Plum), 2-3 ft.............35

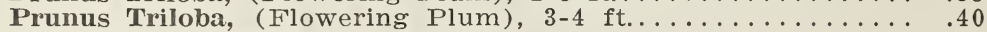

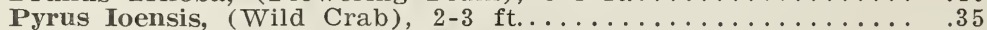

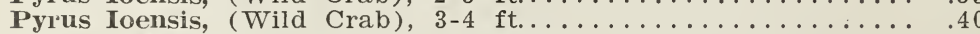

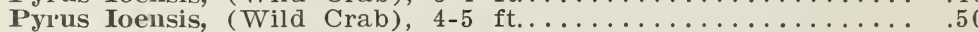

Pyrus Ioensis Fl. Pl., (Bechtels Crab), $18-24$ in........... .40

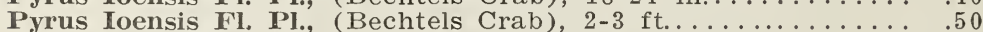

Pyrus Ioensis 1. Pl., (Bechtels Crab), 3-4 ft............ .60

Pyrus Ioensis Fl. Pl., (Bechtels Crab), $4-5 \mathrm{ft} \ldots \ldots \ldots \ldots \ldots \ldots \ldots . \ldots 1.50$

Ptelia Trifoliata, (Am. Hop Tree), 3-4 ft............. .25

Rhodotyphus Irerroides, (White Kerria), $2-3 \mathrm{ft} \ldots \ldots \ldots \ldots \ldots \ldots$

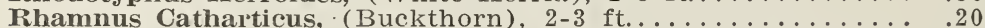

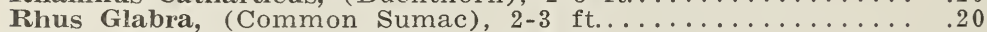

Rhus Glabra, (Common Sumac), $4-5 \mathrm{ft} \ldots \ldots \ldots \ldots \ldots \ldots \ldots \ldots . . \ldots \ldots$

Rhus Typhina Laciniata, (Cut Leaf Sumac), $2-3 \mathrm{ft} . \ldots \ldots . . .20$

Rhus Glabra Laciniata, (Cut Leaf Common Sumac), $12-18$ in... . 20

Rhus Glabra Laciniata, (Cut Leaf Common Sumac), 18-24 in... . .25

Rhus Cotinus, (Purple Fringe), $2-3 \mathrm{ft} \ldots \ldots \ldots \ldots \ldots \ldots \ldots \ldots . . . \ldots$

Rhus Typhina, (Stag Horn Sumac), $2-3 \mathrm{ft} \ldots \ldots \ldots \ldots \ldots \ldots \ldots \ldots . . \ldots \ldots$

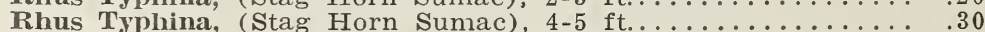

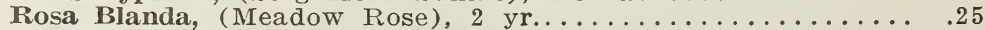

Rosa Nitida, Glossy Swamp Rose), 2 yr.....................

3.00

4.00

35.00

1.25

1.50

2.00

1.00

1.25

1.50

1.50

1.75

2.50

2.25

2.75

2.00

2.00

1.75

2.00

1.50

1.75

2.00

1.75

2.00

2.00

2.00

2.50

2.50

2.50

2.00

2.50

1.75

2.00

3.00

2.50

3.00

3.50

3.00

3.50

4.50

3.50

4.50

5.00

1.50

2.50

1.50

1.50

1.75

1.75

1.50

2.00

1.75

2.50

2.00

2.00
10.00

12.50

18.00

7.00

9.00

11.00

15.00

22.00

20.00

25.00

18.00

15.00

15.00

17.50

12.00

15.00

17.50

15.00

17.50

17.50

17.50

20.00

15.00

17.50

25.00

20.00

40.00

12.00

15.00

20.00

18.00

18.00 


\section{Young Stock for Lining Out}

瞄

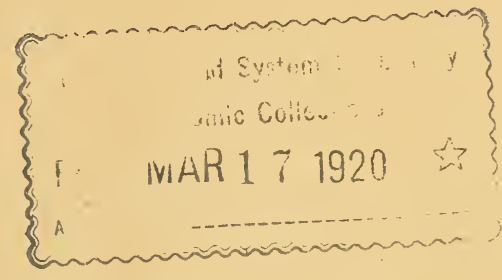

\section{AURORA NURSERIES}

\section{AURORA, ILLINOIS}

Our Trade List of larger Nursery Stock and Perennials will be ready in January. Most items are scarce and prices must be higher at retail. Write us about your requirements. 


\section{CUTTINGS-SEEDLINGS-TRANSPLANTS}

We have finished our grade and count of Young Stock. The list follows.

These plants are in storage and ready for shipment. Unless specified all orders will be shipped by express. All orders will be booked in rotation and filled in the same manner. Preference will be given to purchasers who order a general lot of stock instead of one or two items.

Terms are cash with order, or to those with established credit with us, 30 days less 2 per cent 10 days. Unless you have a credit here please furnish references.

Packing at cost or packed free if cash accompanies order.

\section{C.-Cuttings. S.-Secollings. Tr.-Transplant.}

1000

118

2325

13200

17200

7800

10300

4600

1650

1660

100

4150

400

980

1650

$\mathbf{3 1 7 0}$

4081

2293

160

97

950

123

4025

400

570

265

5100
$\mathbf{7 5 0 0}$

7500
200

37
017

3017

13
069

1267

500

118

12300

3500

2400

1400

18

60

16
12

12

118

28

750

178

5525

208:

134

1011

500

462

114

5000

4800

200

710

$81 !$

Ironia Nigra (Black Chokeberry) 2 yr. tr...

Impelopsis Engelmani (Engelmans Iry) C. .

Impelopsis Quinquefolia (Virginia Creeper)

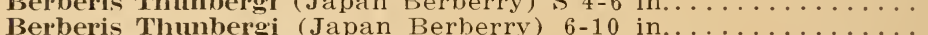

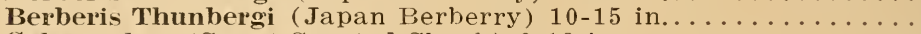

Calycanthus (Sweet Scented Shrub) $6-12$ in. . . . . . . . . . . . . . . . . . . . . . . .

Cornus Stolonifera (Red Osier) S. 10-15 in...

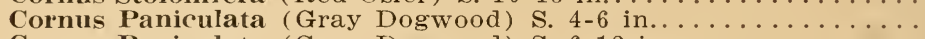

Cornus Paniculata (Gray Dogwood) S. 6-12 in.

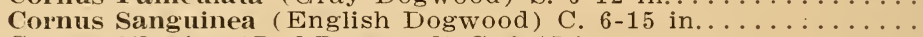

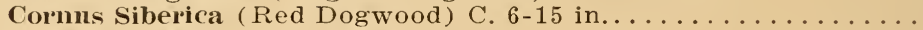

Cornus Sericea (Silky Dogwood) C. 6-12 in.

Cornus Lutea (Golden Bark Dogwood) C. 6-15 in... . . . . . . . . . .

Cotoneaster Acutifolia (See Special Circular).

Celastrus Scandens (American Bittersweet) S. $6-15$ in.........

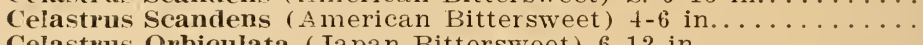

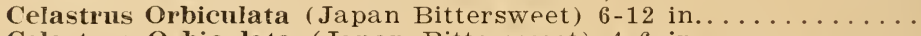

Celastrus Orbiculata (Japan Bittersweet) $4-6$ in.....................
The above vine is a very attractive plant. The growth is slower than the American Bittersweet and the fruit yellow. It is a valuable vine.

Cephalanthus Occidentalis (Button Ball Bush) C. 6-15 in......

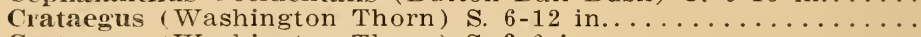

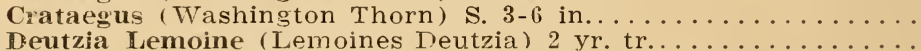

Deutzia Pride of Rochester (Large Flower) C. $6-15$ in..........

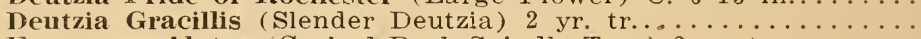

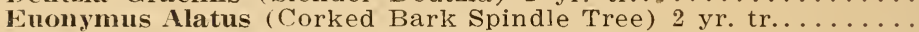

Forsythia Suspensa (Drooping Golden Bell) 2 yr. tr............

Forsythia Intermedia (Golden Bell) C. $6-15$ in..............

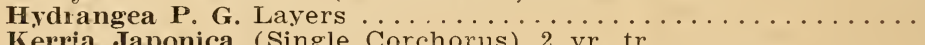

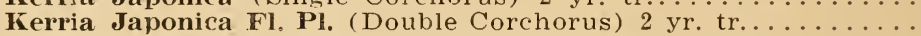

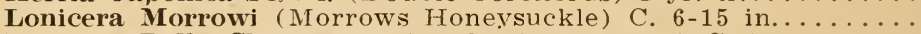

Lonicera Bella Chrysantha (Bush Honeysuckle) C. lyr.........

Ionicera Bella Albida (White Honeysuckle) C. $8-15$ in........

Lonicera Grandiflora (Pink Honeysuckle) C. $6-15$ in..........

Lonicera Scarlet Trmmet Honeysuckle

Ligustrum Leucocarpum (Yellow Fruited Privet) C...........

Ligustrum California (California Privet) C. $6-10 \mathrm{in} . . . \ldots \ldots \ldots$

Ligustrum Ibota (Ibota Privet) 4-6 in..

Ligustrum Ibota (Ibota Privet) $8-15$ in..

Ligustrum Ibota (Ibota Privet) 18-24 in.

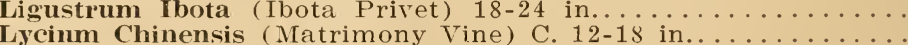

Pyrus Scheideckeri Grafts.

Pyrus Parkmanii Grafts.

Pyins Neidzwetkyana Grafts.

Pyruis Bechte's Dhl, Fl. Grafts.

Pyrus Spertabilis Grafts.

Pyrus Corona Grafts.

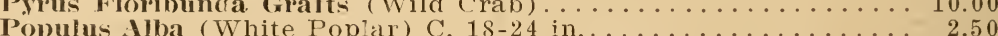

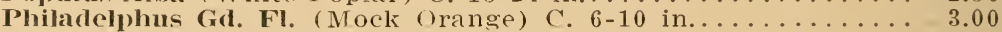

Philadelplus Vivalis (Mock Orange) C $6-15$ in ................ 3.00

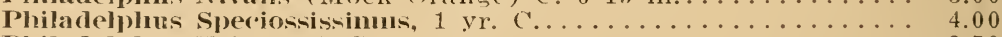

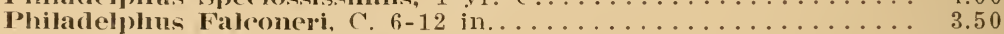

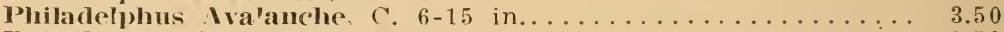

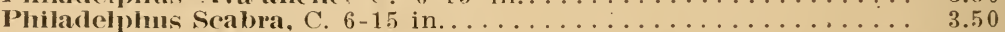

Philadelplus Coronarius (Sweet Scented) C. $6-15$ in.......... 3.50

Philadelphus Corouarius Dwf. (Sweet Scented) 2 yr. tr......... 4.00

Rhus Aromatica (Fragrant Sumac) S. $6-12$ in ............ 5.00

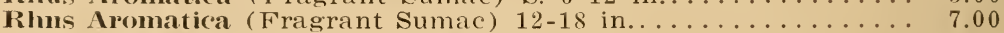

Rosa Blanda tr. (Meadow Rose) .................... 3.00

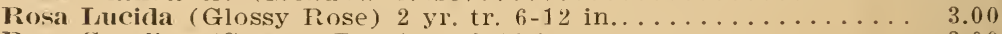

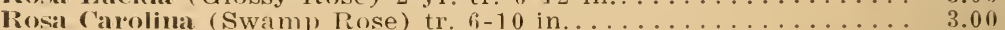

25.00

25.00

12.50

20.00

30.00

22.00

25.00

25.00

30.00

30.00

25.00

35.00

25.00

15.00

25.00

15.00

.00
.50

3.00
5.00

3.00
6.00

8.00
3.50

3.00

.50

4.50
.50

3.00

3.50

2.00

1.50

2.50

3.50
3.50

0.00

0.00
0.00

0.00

0.00

.00

02.00
$0 \quad 000$

28.00

30.00

30.00

30.00

30.00

45.00

65.00

25.00

25.00 
450 Rosa Rubiginosa (Sweet Briar) S. $8-15$ in................ 3.00

75 Ribes Alpimm (Mountain Currant) 1 yr. C................. 4.00

235 Rhodotyphus Kerroides (White Kerria) 2 yr. tr............. 3.50

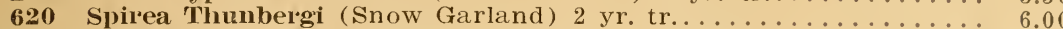

9600 Spirea Van Houtei-(Bridal Wreath) C. $6-15$ in............. 3.50

550 Spirea Rosea (Pink Spirea) 2 yr. tr..................... 5.00

980 Spirea Froebelli (Frobel's Spirea) 2 yr. tr................. 5.00

50 Spirea Sorbifolia, 6-10 in. (Ash Leaf Spirea) . . . . . . . . . . . . 4.00

595 Spirea Marguerite (Dwarf Spirea) 2 yr. tr.................. 5.00

3134 Spirea Salicifolia (Willow Leaf Spirea) C. lyr................. . . . . . . . . . . . .

50 Spirea Wallufi (Red A. W.) 2 yr. tr.................... 10.00

232 Spirea Richmensis (Large Pan Pink) 2 yr. tr.................. . . . . . . . . . . . .

40 Spirea Douglassi (Silver Leaf) C. 1 yr................... 3.00

40 Spirea Nobelaena, C. 1 yr.......................... 3.00

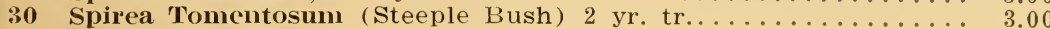

1150 Spirea Opulifolia (Nine Bark) C. 6-15 in................. 3.00

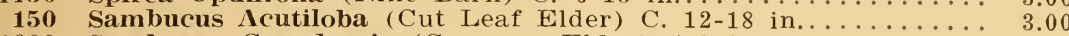

4600 Sambucus Canadensis (Common Elder) 4-8 in. S.......... 2.00

4725 Sambucus Canadensis (Common Elder) $8-16$ in. S............. 2.50

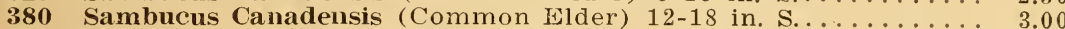

480 Symphoricarpus Glomeratus (Snowberry Compact) $\mathrm{C}$. $6-12$ in. 3.00

1770 Symphoricarpus Vulgaris (Red Snowberry) C. 6-15 in. ....... 3.00

9900 Symphoricarpus Molle (Improved Snowberry) C. 6-12 in....... 3.50

The-above plant is a decided improvement of the White Snowberry. The berries are larger and the foliage is held until frost.

780 Salix Wisconsoni (Weeping Willow) C. $12-18$ in............

534 Sa!ix Aurea (Golden Willow) C. $6-15$ in. . . . . . . . . . . . . . 2.50

690 Salix Britensis (Red Willow) C. $8-12$ in.................. 3.00

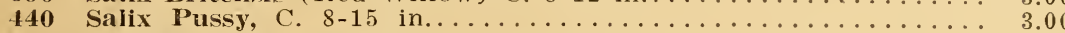

3550 Syringa Persica (Persian Lilac) C. 6-12 in..................6.00

4000 Sylinga Rothomagensis (Red Persian) C. $6-12$ in................ 6.00

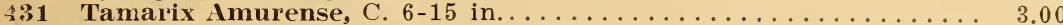

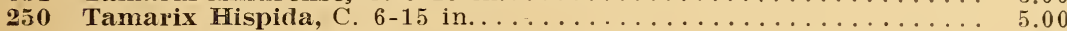

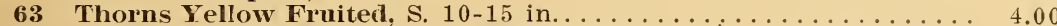

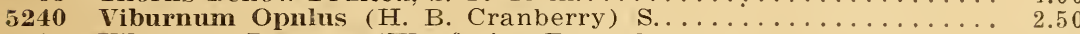

80 Viburuum Lantana (Wayfaring Tree) 2 yr. tr.............. 6.00

257 Viburnum Opulus Sterilis (Snowball) 2 yr. tr............ 5.00

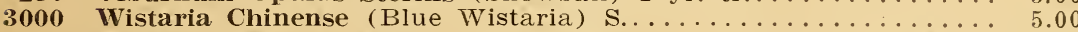

18 Weigela Lutea (Yellow) C. $8-15$ in. ................. 5.00

50 Weigela Desboise (Deep Rose) C. $6-10$ in.................. 5.00

3450 Weigela Rosea (Pink) C. 6-12 in. ...................... 5.50

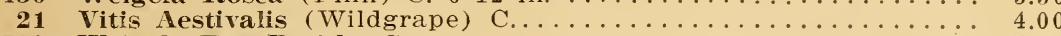

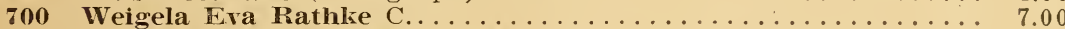

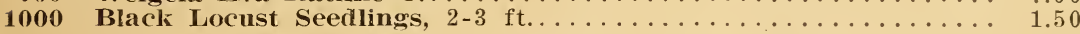

\section{FRAME CUTTING}

\section{Read This Note Carefully}

The following cuttings were rooted in sand during the summer of 1919 , and will be available for shipment about March $15 \mathrm{th}, 1920$. We are wintering these cuttings in order to put them into the hands of the buyer in good condition.

We recommend planting most varieties in beds, although if properly and carefully handled they will do well in the field.

We will book orders for these cuttings until February 20th, 1920, at which time orders will be filled by giving to each buyer his proportion of the cuttings according to the quantity ordered.

Orders received after that date will be subject to count after former orders have been filled.

Packing at cost, or packed free if cash accompanies orders.

100

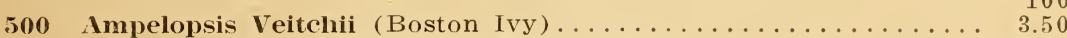

500 Ampelopsis Quinquefolia (Virginia Creeper) ............. 2.50

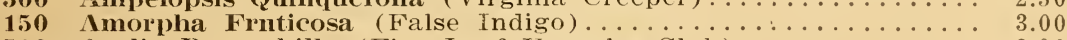

500 Iralia Pentyphilla (Five Leaf Hercules Club) .............. 3.00

1000 Cornms Mascula (Cornelian Cherry).................... 3.00

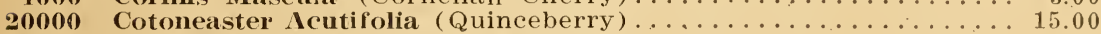

500 Deutzia Lemoine (Lemoines Deutzia) . . ... . . . . . . . . . . 3.00

400 Dentzia Gracillis (Slender Deutzia) . . . . . . . . . . . . . . 3.00

2000 Dentzia Pride of Rochester (Large Flowered) . . . . . . . . . . . . . 2.50)

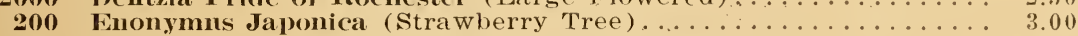

3000 Enonymus Alatus (Corked Barked Spindle Tree) ........... 4.00

1500 Enonymus Eumopea (English Strawberry Tree) . . . . . . . . . . . 3.00

3000 Forsythia Suspensa (Drooping Golden Bell) . .............. 3.00

3000 For'sythia Fortunei (Fortune's Golden Bell) . . . . . . . . . . . . 3.00

5000 
Lonirera Ledebori (Honeysuckle) ...................

Lonicera Maacki (New Honeysuckle) . . . . . . . . . . . . . . . .

Philadelphus Lemoine Erectus (Dwarf Syringa) ... 3.00

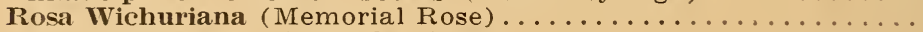

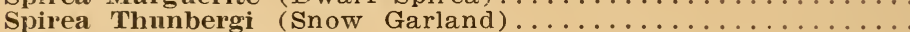

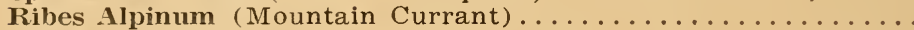

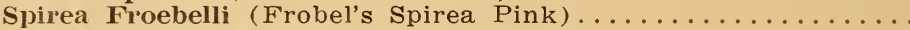

Spirea Anthony Waterer (Dwarf Pink).................

Spirea Collosa

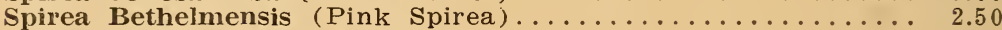

Spirea Sorbifolia (Ash Leaf Spirea) ...................

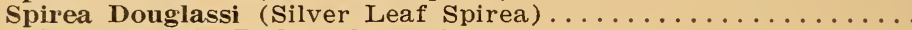

3.00

Spirea Wallufi (Red Anthony Waterer).................

1000

Spirea Opulifolia Aurea (Yellow Leaf Spirea)..............

3.00

3.00

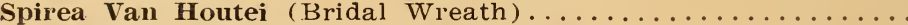

3000

18000

1000

6000

2500

1500

1000

2000

4500

1800

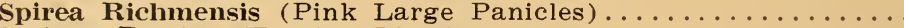

3.00

2.50

3.50

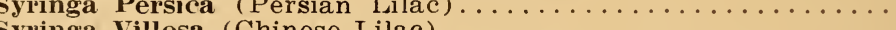

3.50

3.50

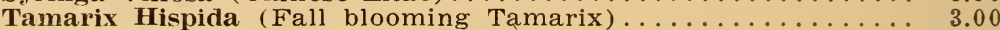

Viburnum Opulus Nana (Dwarf H. B. Cranberry) ...........

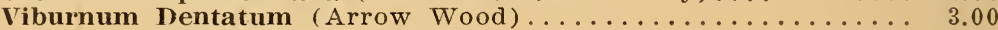

Viburnum Cassanoides (White Rod) ................. 3.50

Viburnum Lantana (Wayfaring Tree) . . . . . . . . . . . . . 3.50

Viburnum Molle (Shiny Viburnum) . . . . . . . . 50

Viburnum Opulns $(H$. B. Cranberry) ................. 3.00

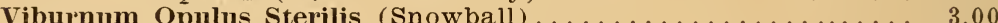

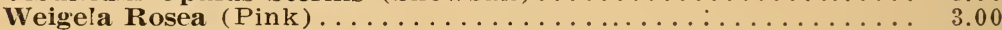

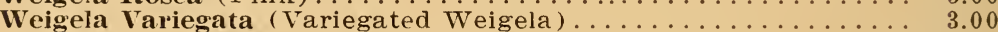

Red Raspberry, assorted varieties.

8000 Red Raspberry, St. Regis.

22000 Blackberries, assorted varieties.

2000 Raspberry Golden Queen.

500 Currant, Red Cross, 2 yr. No. 1

500 Currant, Fays, 2 yr. No. 1

300 Currant, Perfection, 2 yr. No. 1.

200 Currant White Grape, 2 yr. No. 1.

100 Currant, Black Champion, 2 yr. No, 1

200 Grape, Moore's Diamond.

500 Tritoma.

500 Gooseber'y, Downing.

1000 Hydrangea' P. G., 12-18 in

1000 Hydrangea P. G. $18-24$ in.

1000 Hydrangea $P . G, 2-3 \mathrm{ft}$.

If unable to do the varieties called for, quote on what you can do.

\section{SEEDS}

The following seeds are offered for immediate sale

20 lbs. Rosa Setigera $\$ 1.00$ per lb.

10 lbs. Amalanchier Botry apium $\$ 1.50$ per $1 \mathrm{~b}$.

5 lbs. Eleagnus Longipes $\$ 1.25$ per $1 \mathrm{~b}$.

5 lbs. Viburnum Dentatum $\$ 1.00$ per ib.

50 lbs. Rosa Rubiginosa $\$ 1.00$ per lb.

50 lbs. Russian Olive $\$ 1.25$ per $1 b$.

10 lbs. Prunus Americana $\$ 1.50$ per $1 \mathrm{~b}$.

10 lbs. Crataegus Crus Galli $\$ 1.25$ per ib.

3 lbs. Prunus Serotina $\$ .75$ per $1 b$.

25 lbs. Rosa Rugosa $\$ 1.00$ per lb.

100 lbs. Crateagus Cordata (Stratified 1918) $\$ .60$ per $1 b$.

10 lbs. Ampelopsis Veitchii $\$ 2.00$ per $1 \mathrm{~b}$.

3 lbs, Celastrus Scandens $\$ .75$ per $1 \mathrm{~b}$.

2 bu. Wild Crab Apple (Corona) whole apples $\$ 3.00$ per bu. 


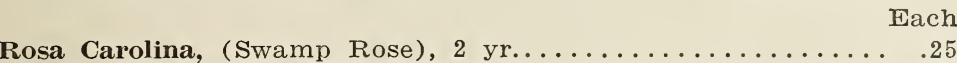

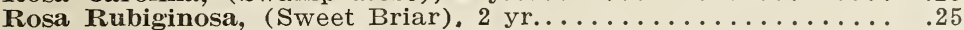

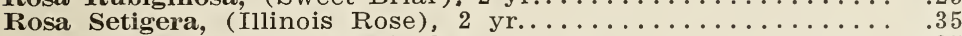

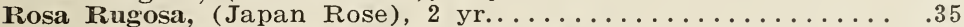

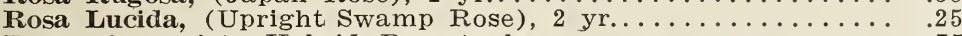

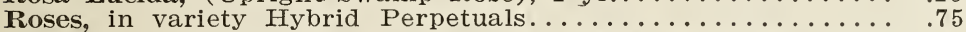

Mrs. John Laing, Paul Neyron, Baroness Rothchild, Baron Bonstettin, Frau Karl Druski, General Jack, John Hopper, Magna Charta, M. P. Wilder, Prince Camille DeRohen, Fisher Holmes, Persian Yellow.

Roses, Hybrid Tea in variety..............................

Roses, Baby Ramblers, in variety, Red, White, Pink........

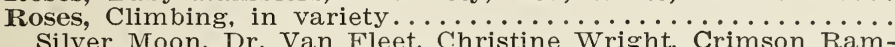
Silver Moon, Dr. Van Fleet, Christine Wright, Crimson Ram-
er, Dorothy Perkins, Dorothy Perkins White, Flower of Fairbler, Dorothy Perkins, Dorothy Perkins

Ribes Aurem, (Yellow Flowering Currant), 3-4 ft...........30

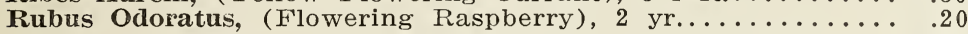

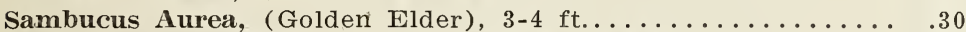

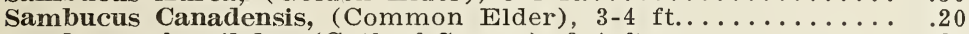

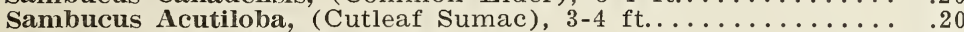

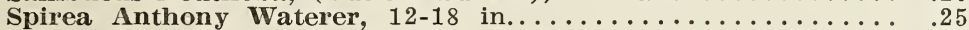

Spirea Aurea, (Golden Spirea), $3-4 \mathrm{ft} \ldots \ldots \ldots \ldots \ldots \ldots \ldots \ldots \ldots . . \ldots \ldots$

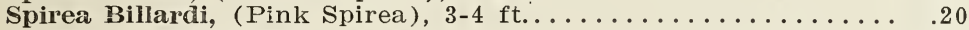

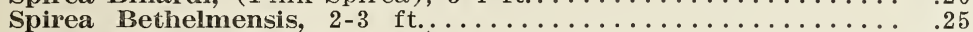

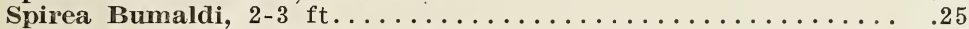

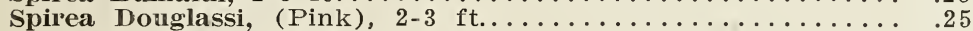

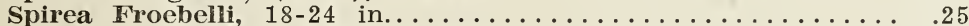

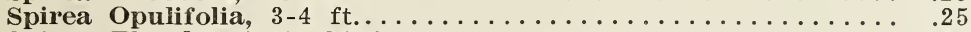

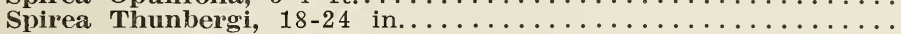

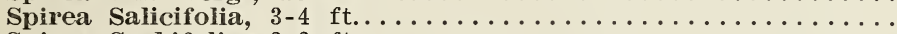

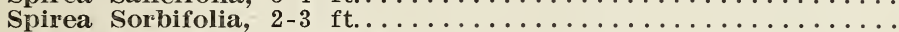

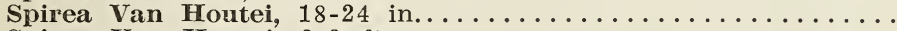

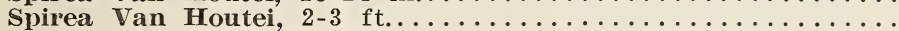

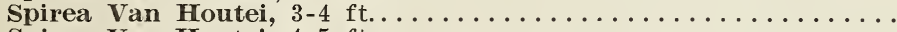

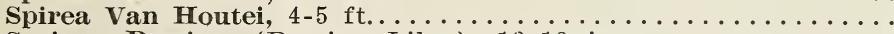

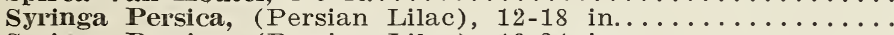

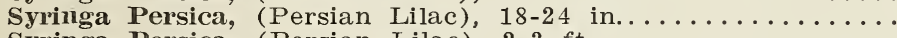

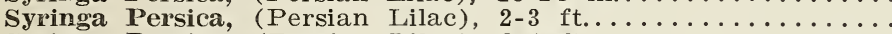

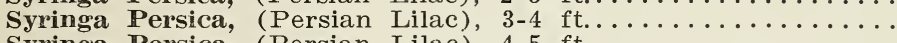

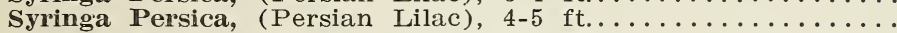

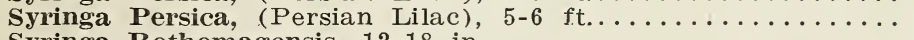

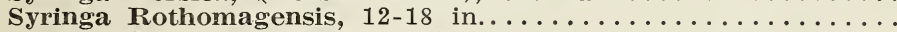

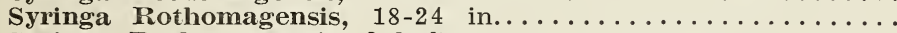

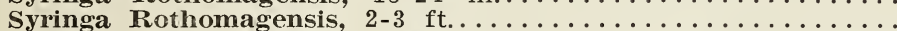

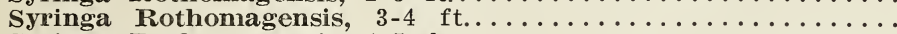

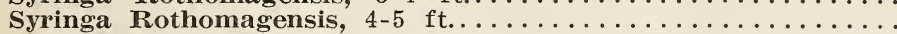

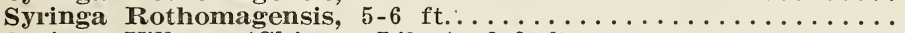

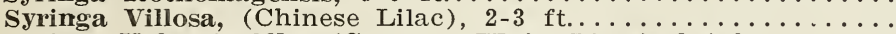

Syringa Vulgaris Alba, (Common White Lilac) $3-4 \mathrm{ft} . \ldots \ldots \ldots$.

Syringa Vulgaris, (Common Purple Lilac), $2-3 \mathrm{ft} . \ldots \ldots \ldots \ldots$

Syringa Vulgaris, (Common Purple Lilac), $3-4 \mathrm{ft} . . . \ldots \ldots \ldots$.

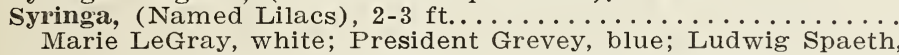

dark red; Alfonse Lavelle, double blue; Frau Dammann, white; Emil Lemoine, double pink; Chas. X, reddish purple.

Symphoricarpus Glomeratus, (Compact Coral Berry), 2-3 ft...

Symphoricarpus Molle, (Improved Snowberry), 2-3 ft.........

Symphoricarpus Racemosus, (White Snowberry), $2-3 \mathrm{ft} . . .$. .

Symphoricarpus Vulgaris, (Red Snowberry), $2-3$ ft.........

Symphoricarpus Vulgaris, (Red Snowberry), 3-4 ft. . . . . . . . .

Tamaris Amurense, $3-4$ ft. . . . . . . . . . . . . . . . .

Vilburnum Dentatum, (Arrow Wood), $2-3 \mathrm{ft} . \ldots \ldots \ldots \ldots$

Viburnum Opulus, (High Bush Cranberry), $2-3 \mathrm{ft} . \ldots \ldots \ldots \ldots$. . . . .

Viburnum Opulus, (High Bush Cranberry), $3-4 \mathrm{ft} \ldots \ldots \ldots \ldots \ldots$
Viburnum
Opulus Sterilis, (Snowball), $3 \mathrm{ft} . \ldots \ldots \ldots \ldots$

Viburnum Lantana, (Wayfaring Tree), $2-3 \mathrm{ft} . \ldots \ldots \ldots \ldots \ldots$

Weigela Amabilis, $2-3 \mathrm{ft} . \ldots \ldots \ldots \ldots \ldots \ldots \ldots \ldots \ldots \ldots$

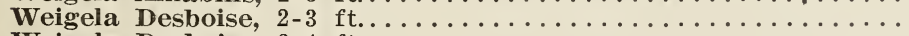

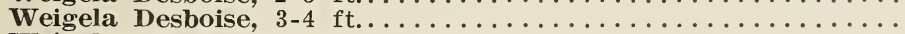

Weigela Rosea, $2-3$ ft. . . . . . . . . . . . . . . . . .

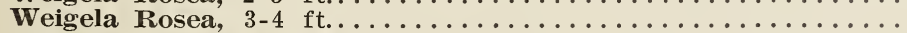

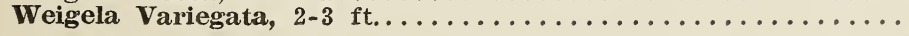

10

2.00

2.00

3.00

3.00

2.00

7.00

100

18.00

18.00

25.00

25.00

18.00

7.00

7.00

7.00

2.25

1.75

2.50

1.75

1.75

2.25

3.00

1.50

1.75

1.75

2.00

1.75

2.50

1.50

2.00

1.25

1.75

3.00

3.50

1.50

1.75

3.50

4.25

6.00

1.50

1.75

3.00

4.50

6.00

3.00

2.50

1.75

2.00

4.00

15.00

17.50

35.00

17.50

10.00

15.00

25.00

30.00

12.00

15.00

12.00

15.00 


\section{VINES}

\begin{tabular}{|c|c|c|c|}
\hline & Each & 10 & 100 \\
\hline 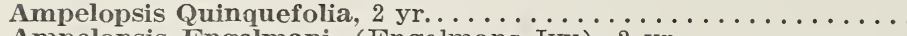 & .20 & & \\
\hline Ampelopsis Engelmani, (Engelmans Ivy), 2 yr........... & .20 & 1.75 & 15.00 \\
\hline 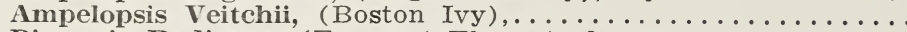 & .50 & & \\
\hline 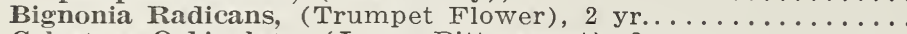 & .20 & 1.75 & 15.00 \\
\hline 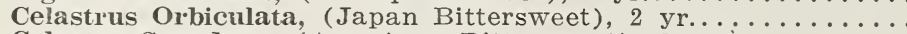 & .20 & 1.75 & 15.00 \\
\hline Celastus Scandens, (American Bittersweet), 2 yr........... & .20 & 1.75 & 15.00 \\
\hline 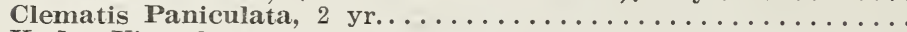 & .30 & 2.50 & \\
\hline 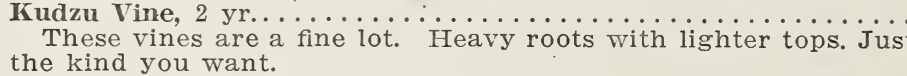 & .20 & 2.50 & 20.00 \\
\hline Lycium Chinensis, (Matrimony Vine) $\ldots \ldots \ldots \ldots \ldots \ldots \ldots$ & .20 & 1.75 & 15.00 \\
\hline Lonicera Halleana, (Honeysuckle Vine)... & .20 & 1.75 & \\
\hline Lonicera Sempervirens, (Scarlet Trumpet) .............. & .20 & 1.75 & \\
\hline 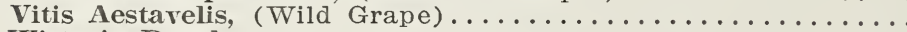 & & & \\
\hline Vistaria Purple ................. & & & \\
\hline
\end{tabular}

\section{EVERGREENS.}

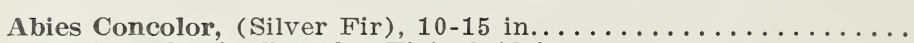

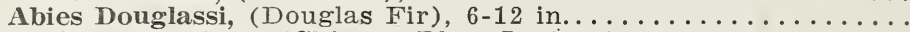
Juniperus Stricta, (Chinese Blue Juniper), 6-12 in......... Juniperus Stricta, (Chinese Blue Juniper), $12-15$ in........... Juniperus Hibernica, (Irish Juniper), $2-3 \mathrm{ft} . \ldots \ldots \ldots \ldots \ldots \ldots$

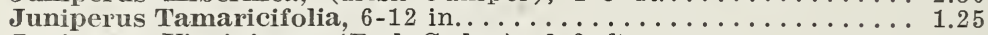
Juniperus Virginiana, (Red Cedar), $2-3$ ft...............60

Pinus Strobus, $8-15$ in. (White Pine), twice tr............

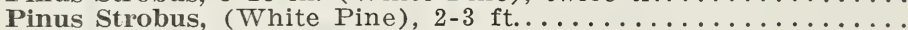

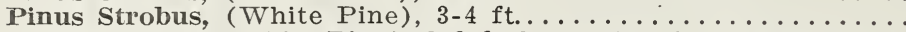

Pinus Strobus, (White Pine), 2-3 ft. heavy Specimens, solid balls

Pinus Strobus, (White Pine), 3-4 ft. heavy Specimens, solid balls 2.25

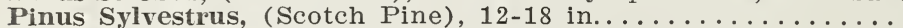

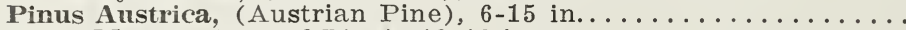

Pinus Mugho, (Dwarf Pine), $12-18$ in. . . . . . . . . . . . . . .

Picea Canadensis, (Black Hill Spruce), 6-12 in............

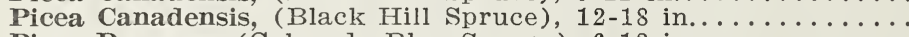

Picea Pungens, (Colorado Blue Spruce), 6-12 in............

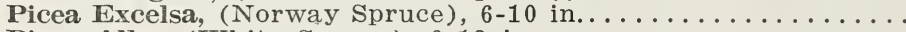

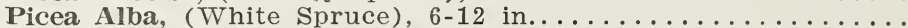

Thuya Pyramidalis, (Pyramidal Arbor Vitae), 8-12 in........

Thuya Pyramidalis, (Pyramidal Arbor Vitae), 12-18 in.......

Thuya Occidentalis, (American Arbor Vitae), 12-18 in.........

Thuya Occidentalis, (American Arbor Vitae), 18-24 in........

Thuya Occidentalis, (American Arbor Vitae), 2-3 ft..........

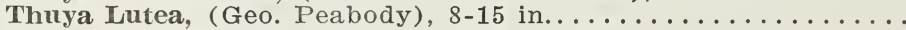

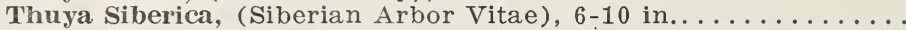

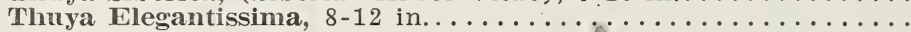

\section{PERENNIALS}

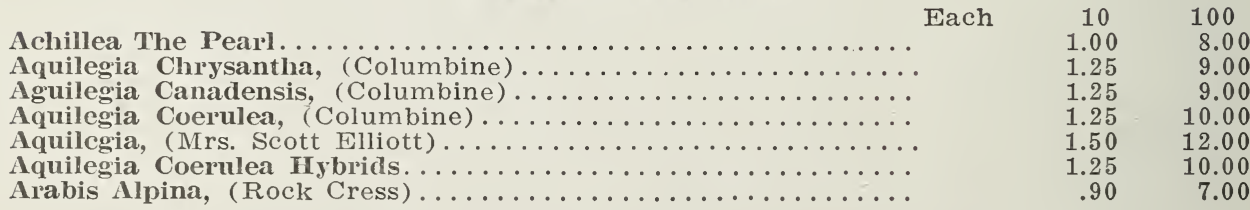

\section{WEIGELA ROSEA}

Plants of this variety are scarce. We have a few light 2-3 ft. plants, not heavy enough for our regular grade. They are, however, very fine little plants. Price, 20 cents each, $\$ 1.75$ per $10, \$ 15.00$ per 100 . 


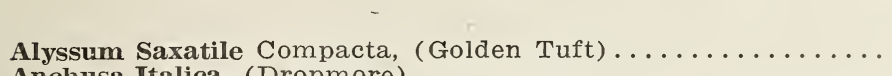

Each

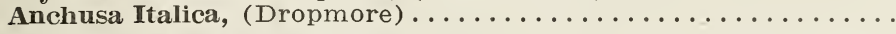

Boltonia Latisquama, (False Chamomile) ................

1.50

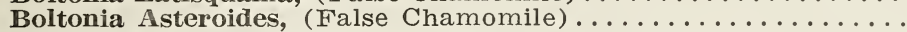

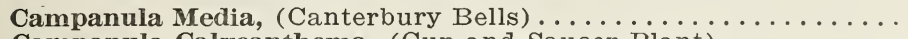

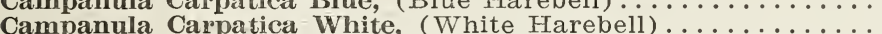

Campanula Persicifolia BIue, (Peach Leaved Canterbury Belli)...

Campanula Persicifolia White, (Peach Leaved Canterbury Bell)

1.25

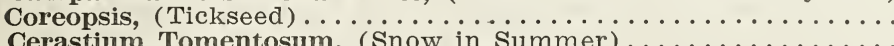

1.25

1.00

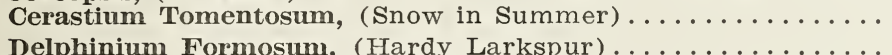

1.25

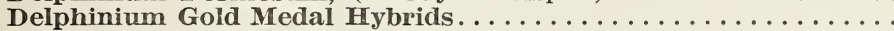

Delphinium Chinensis.

Delphinium Belladona. . . . . . . . . . . .

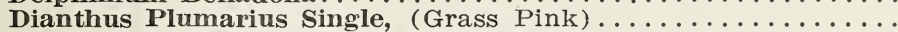

Dianthus Plumarius Double, (Grass Pink) . . . . . . . . . . . .

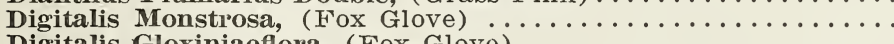

Digitalis Gloxiniaeflora, (Fox Glove) ................

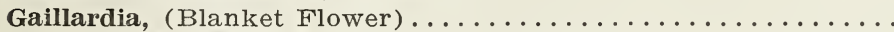

Gypsophilla Repens, (Creeping Baby's Breath) ..............

Gypsophilla Paniculata, (Baby's Breath) ...............

Hemerocallis Flava, (Yellow Day Lily).

Helenium Autumnale, (Sneeze Wort).

Helenium Riverton Gem, (Sneeze Wort) . . . . . . . . . . . .

Hibiscus Crimson Eye, (Mallows).

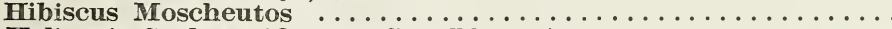

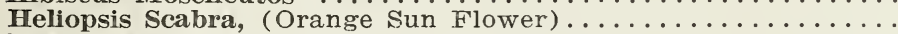

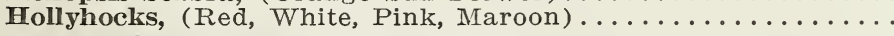

Iberis Sempervirens, (Hardy Candytuft) . . . . . . . . . . .

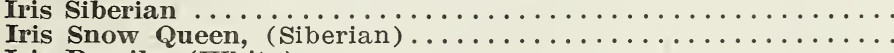

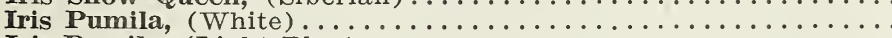

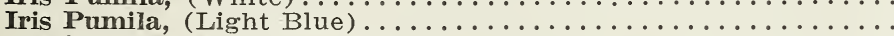

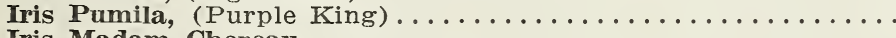

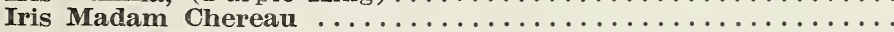

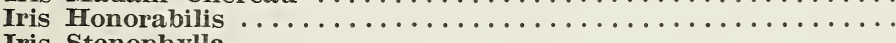

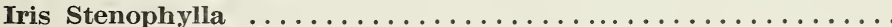

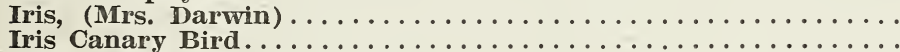

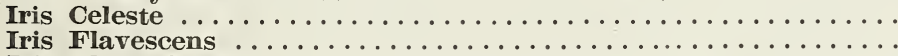

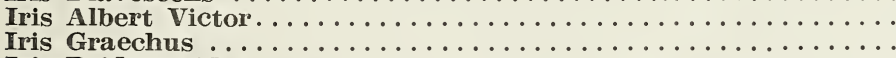

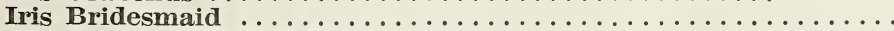

Iris Palida Delmatica. . . . . . . . . . . . . . . . . . . .

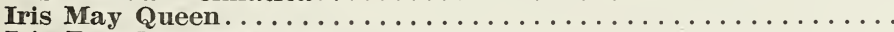

Iris Pseudoacorus . . . . . . . . . . . . . . . . . . .

Lobelia Syphilitica, (Cardinal Flower) $\ldots \ldots \ldots \ldots \ldots \ldots \ldots$

Lathyrus Latifolius, (Hardy Sweet Pea) ................

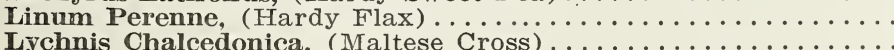

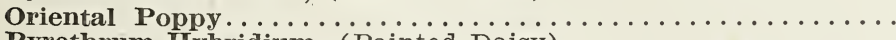

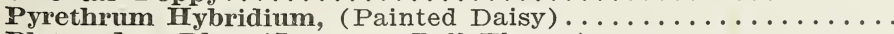

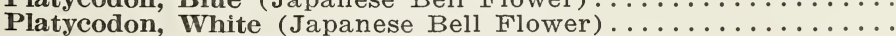

1.00

1.00

Pentstemon Barbatus, (Beards Tongue) $\ldots \ldots \ldots \ldots \ldots \ldots \ldots$

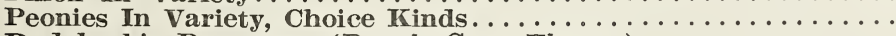

Rudebeckia Purpurea, (Purple Cone Flower) ..............

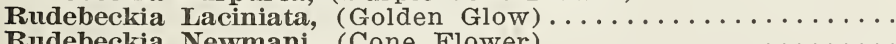

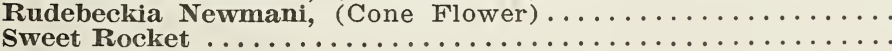

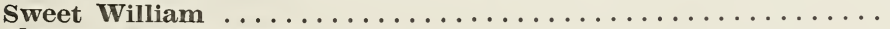

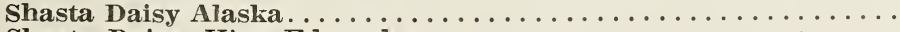

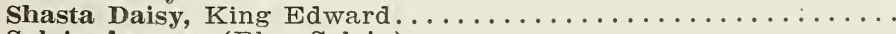

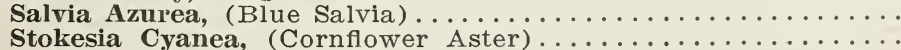

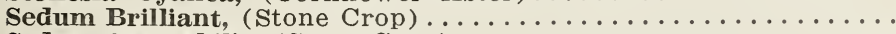

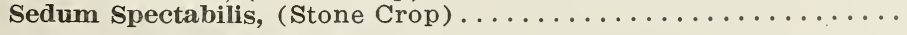

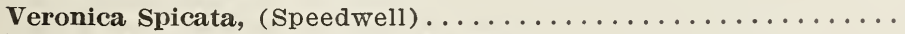

Yucca Filamentosa, (Adams Needle)................... 


\section{FRUITS}

Each $\quad 10 \quad 100$

Apples, $\frac{11}{16}$ in., $5-6 \mathrm{ft}$. in variety .................... $1.25 \quad 10.00$

Summer-Duchess, Yellow Transparent, Eatly Harvest.

Fall-Red Astrachan, Sweet Rough, Wealthy.

Winter-Salome, Fameuse, Ramsdell Sweet, McIntosh Red,

Jonathan, Grimes Golden, Minkler, Willow Twig, Talman Sweet,

N. W. Greening, Staymens Winesap, Black Ben Davis, Mammoth

Black Twig, Baldwin, Northern Spy, Delicious.

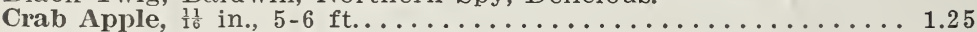
Early Strawberry, Hyslop, Whitney, Transcendent.

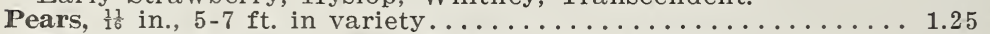
Bartlett, Clapps, Favorite, Howell, Flemish Beauty, Duchess, Keiffer.

Peaches, $\frac{9}{16}$ in., $5 \mathrm{ft}$. in variety................. 1.00 Elberta, Champion.

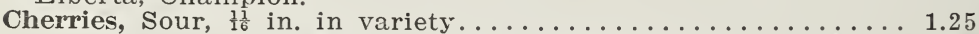

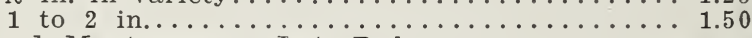
Early Richmond, Montmorency, Late Duke.

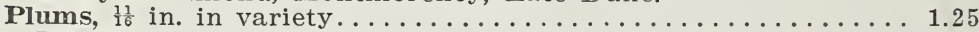
Wyant, Surprise, Hammer, Sapa, Hanska, Opata.

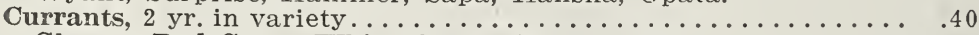
Cherry, Red Cross, White Grape, Pomona, etc.

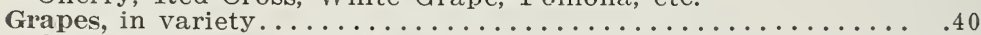
Concord, Agawam, Niagara, etc.

Gooseberries, in variety........................ .50 Houghton, Downing, Red Jacket.

Blackberries, in variety....................... .20 Erie, Snyder, Eldorado, etc.

Raspberries Red, in variety.................... .15 Cuthbert, Miller, Loudon, King, etc.

Raspberries, St. Regis......................... . 20

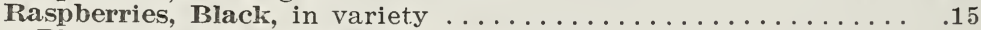
Plum, Farmer, Kansas, Cumberland, etc.

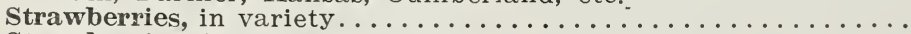

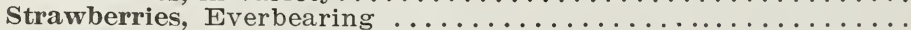

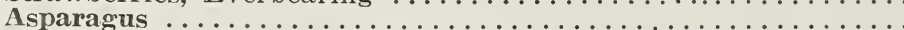

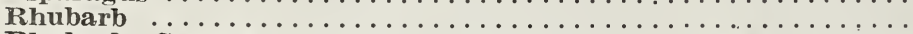

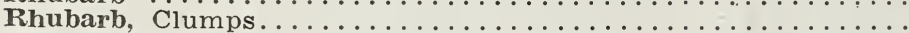

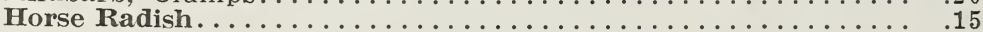

10.00

10.00

11.00

30.00

1.50

12.00

1.25

10.00

1.50

12.00

$1.25 \quad 10.00$

1.50

2.00

1.25

1.00

1.50

1.25

\section{SEEDS}

The following seeds are offered for immediate sale:

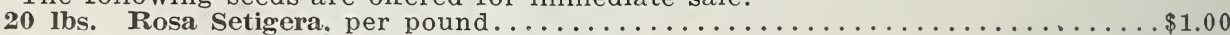

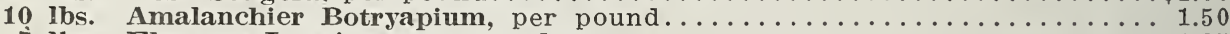

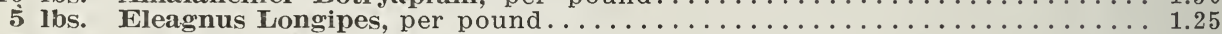

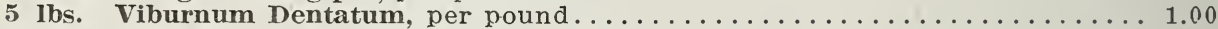

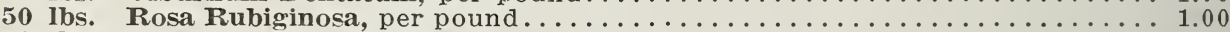

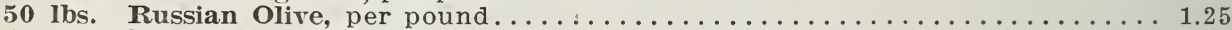

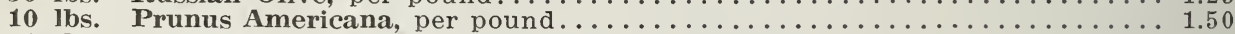

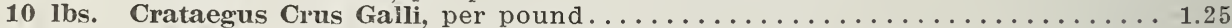

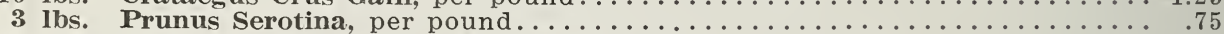

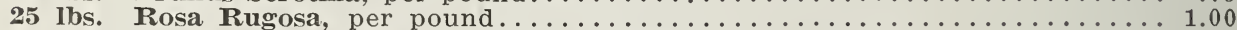

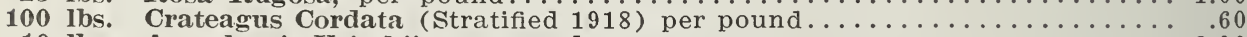

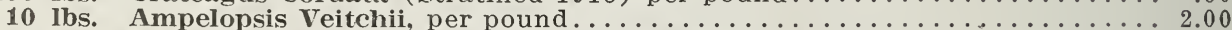

2 bu. Wild Crab Apple (Corona) whole a pples, per bushel ......................

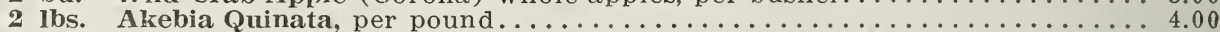

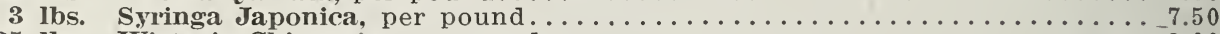

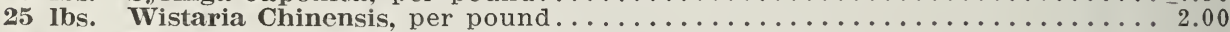

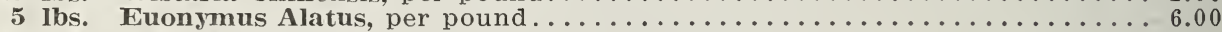

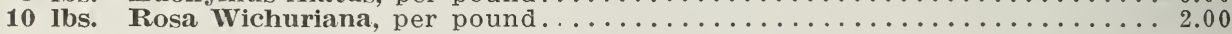

\section{KOSTERS BLUE SPRUCE, 18-24 inch . . \$3.00 each} KOSTERS BLUE SPRUCE, 2-3 ft. . . . . 4.00 each 